\title{
Depth Distribution of Ge Fraction in Very-Thin-SGOI Layers Using Total-External-Reflection X-ray Diffraction
}

\author{
T. Kawamura ${ }^{1}$, H. Omi, M. Mizumaki* and S. Kimura* \\ NTT Basic Research Labs, NTT Corporation, 3-1, Wakamiya, Morinosato, Atsugi, Kanagawa 243-0198, Japan \\ Fax: 81-46-240-3362, e-mail: homi@will.brl.ntt.co.jp \\ * Japan Synchrotron Radiation Research Institute, 1-1-1, Kouto, Sayo-cho, Sayo-gun, Hyogo 679-5198 Japan
}

\begin{abstract}
Very thin silicon-germanium-on-insulator (SGOI) substrates have been investigated using the total-external-reflection $x$-ray diffraction technique (TER-XD). The results show that the Ge segregation layer was formed at the interface between the oxidization layer and the SGOI layer. In-plane diffraction using TER-XD also shows that the Ge portion decreased with increasing incident angles of x-rays, suggesting the inhomogeneity of Ge distribution in the SGOI layers. These results are consistent with the previous work concerning to the Ge oxidization, suggesting additional annealing procedure will be required to increase crystalline quality of SGOI layers. Key words: GIXD, SGOI, total-reflection, in-plane x-ray diffraction, total-external-reflection
\end{abstract}

\section{INTRODUCTION}

Silicon-germanium-on-insulator (SGOI) has been aggressively investigated in the last decade since it works as a virtual substrate [1] for strained-silicon devices, which overcome the physical limitations of silicon-based metal-oxide-semiconductor field-effect transistors (MOSFETs) [2,3]. Combining SGOI with strained silicon has additional advantages, such as reductions of the body effect and parasitic junction capacitance [4-6]. Further improvement of device performance can be achieved by fabricating devices on SGOI substrates less than several ten-nanometers thick and operating them in the fully depleted mode [7]. Several techniques have been used to fabricate SGOI substrates, such as ion-implantation into a SiGe layer, wafer bonding, and annealing SiGe on silicon-on-insulator (SOI) wafers [8-11]. To improve the crystalline quality of SGOI layers with a higher Ge fraction, Tezuka et at. have proposed a unique SGOI fabrication process based on Ge condensation through oxidization of SiGe layers on SOI [12], which can decrease the formation of dislocations.

Although several SGOI substrates have been fabricated, stress control in SGOI layers still remains a crucial problem in obtaining good-quality SGOI substrates. This problem is more serious for thinner SGOI layers since Bragg intensities in the reciprocal space maps (RSMs) should be weaker, causing decrease of accuracy to determine the in-plane and out-of plane strains.

Raman spectroscopy [13] and x-ray diffraction [14] techniques have been commonly used to evaluate residual strains and Ge fractions. Raman spectroscopy is easier to use, but more accurate strain information can be obtained using high-resolution x-ray diffraction [15]. For both techniques, the Ge fraction determination is based on Vegard's law [16] but the uniform layers are required to use this law, which are not always satisfied in very thin layers. Recently, total-external-reflection $\mathrm{x}$-ray diffraction (TER-XD) has been demonstrated as a depth-selective real structure analysis $[17,18]$, which uses total reflection effect of $x$-rays close the critical angle. In this article, we describe the theoretical bases of the TER-XD technique and the results of very-thin SGOI layers analyzed with using this technique.

\section{BASES}

When $\mathrm{x}$-rays impinged on samples near the critical angle, four configurations of the incident and reflected $\mathrm{x}$-rays is considered, as shown in Fig. 1. Here, $\alpha_{\mathrm{i}}, \alpha_{\mathrm{f}}$ and $\alpha_{c}$ are the incident and exit angles of x-rays, and the critical angle determined by the each layered materials, respectively. In the case of $\alpha_{i}<\alpha_{c}$, incident x-rays penetrate into samples within several nanometers to ten-nanometers, and x-ray diffraction from samples reflects sample structure close to surface, obtaining surface-sensitive structural information using this technique (case 1 and 3 in Fig. 1.). If $\alpha_{i}>\alpha_{c}$ (case 2 and 4), incident $\mathrm{X}$-rays penetrate deeply, and Bragg diffractions occur whole region of samples. The penetration depth of which amplitude of x-ray decreases $1 / \mathrm{e}$ is limited only by the absorptions of sample materials. These configurations sometimes are called "grazing incidence x-ray diffraction (GIXD)", and widely used to evaluate sample structures close to the surface region within several nanometers to several micron meters.

However, in the case of $\alpha_{\mathrm{f}}<\alpha_{\mathrm{c}}$, measured region inward surface normal should be considered more precisely since the penetration depth is affected by the total-reflection effect (case 1 and 2 in Fig. 1). This effect has been investigated last decade and formalized as "scattering depth" of Bragg diffraction as follows [17, $18]$;

\footnotetext{
1 Present address: Nichia Corporation.
} 


$$
\begin{aligned}
& \Lambda=\frac{\lambda}{2 \pi\left(l_{i}+l_{f}\right)}, \\
& l_{i, f}=\frac{1}{\sqrt{2}}\left\{\left(2 \delta-\sin ^{2} \alpha_{i, f}\right)+\left[\left(\sin ^{2} \alpha_{i, f}-2 \delta\right)^{2}+4 \beta^{2}\right]^{1 / 2}\right\}^{1 / 2}
\end{aligned}
$$

Here, $\Lambda$ is the scattering depth, and $\lambda, \delta$ and $\beta$ are the wavelength and the real and imaginary part of the reflactive indices of each layer. The scattering depth differs from the penetration depth since it is the region of depth where observed Bragg diffraction occurs. It should be noted that TER-XD occurs at both $\alpha_{i}<\alpha_{c}$ and $\alpha_{i}>\alpha_{c}$, which is different from the ordinal surfacesensitive GIXD technique, and exit-angle resolved measurement is required for TER-XD measurement.

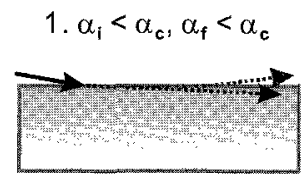

TER-XD Case

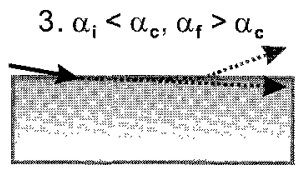

TER-XD Case
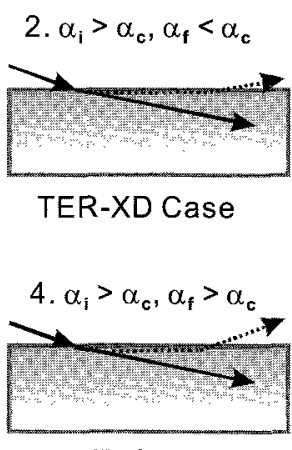

GIXD Case

Fig. 1. Schematic geometries of TER-XD and GIXD layout.

Another factor for TER-XD is the modulated amplitude of $\mathrm{x}$-rays in the total reflection region. This is known as the distorted-Born-wave-approximation (DWBA) [19], and the effect of DWBA to the TER-XD has been also investigated [18]. The Bragg intensity $I\left(Q_{x}, Q_{y}, Q_{z}\right)$ of TED-XD including DWBA effect is described as follows [18];

$$
\begin{aligned}
I\left(Q_{x}, Q_{y}, Q_{z}\right) & \propto\left|T_{i}\right|^{2}\left|S\left(q_{x}, q_{y}, q_{z}\right)\right|^{2}\left|T_{f}\right|^{2} e^{-\sigma^{2} Q_{z}^{2}} . \\
S\left(q_{x}, q_{y}, q_{z}\right)= & \sum_{m=0}^{\infty} e^{-i q_{x} x_{m}} \sum_{n=0}^{\infty} e^{-i q_{y} y_{n}} \times \\
& \int_{0}^{\infty} \chi_{H}\left(q_{z}, z\right) e^{-i q_{z} z_{j}} d z \\
= & G\left(q_{x}, q_{y}\right) \int_{0}^{\infty} \chi_{H}\left(q_{z}, z\right) e^{-i q_{z} z_{j}} d z
\end{aligned}
$$

Here, $T_{i}, T_{f}$ and $S\left(q_{x}, q_{y}, q_{z}\right)$ are the transmission factor of incident and diffracted $x$-rays between the surface and the vacuum, and a scattering factor of the layered-film.

The last term $e^{-\sigma^{2} Q_{2}^{2}}$ of $\mathrm{Eq}$. 2a means the empirical dumping factor that is similar to the surface roughness factor of x-ray reflectivity. The scattering factor depends on in-plane scattering function $G\left(q_{x}, q_{y}\right)$ and electron susceptibilities $\chi_{\mathrm{h}}\left(q_{z}, z\right)$ of each layers. The extinction of incident and exit $x$-rays is included as $e_{z}^{-i q} z$ since $q_{z}$ is imaginary at the total reflection condition without considering absorption effect.

\section{EXPERIMENTAL}

The SGOI samples were fabricated on the SIMOX (Separation by IMplanted OXygen) (001) substrates using flowed processes.

(1) Repeating oxidization and removing $\mathrm{SiO}_{2}$ layers using etching process until the thickness of top Si layers reached to about $20-\mathrm{nm}$.

(2) Evaporating 20-nm $\mathrm{Si}_{1-\mathrm{x}} \mathrm{Ge}_{\mathrm{x}}$ layers after thermal cleaning of the top Si layers.

(3) Oxidizing $\mathrm{Si}_{1-\mathrm{x}} \mathrm{Ge}_{\mathrm{x}} / \mathrm{Si} / \mathrm{SiO}_{2} / \mathrm{Si}$ samples to fabricate SGOI layers sandwiched by $\mathrm{SiO}_{2}$ layers.

A typical structure of samples is shown as the inset of Fig. 2.

All x-ray measurements were performed at the BL46XU beamline of the synchrotron radiation facility SPring-8. To measure TER-XD diffraction intensities, a multi-axes goniometer was used with $0.1127-\mathrm{nm}$ wavelength. A set of vertical slits was used in front of the $\mathrm{NaI}$ detector to set the exit angle at 0.23 degrees which corresponds the critical angle of the SGOI layer. Incident $\mathrm{x}$-rays were collimated as $100 \mu \mathrm{m}$ (vertical) times $200 \mu \mathrm{m}$ (horizontal) using quadric-slits and high-order composition of the beam was removed using a set of Rh-coated-mirror. Since the Bragg diffractions perpendicular to the surface, such as (004) were very weak, (220) diffraction of Si and SGOI layers, which is parallel to the surface, was measured using $\omega-2 \theta$ scan mode with various incident angles.

\section{RESULS AND DISCUSSIONS}

Figure 2 shows the SGOI (220) diffraction profiles along $q_{z}$ direction with various incident angles. The peaks at $\alpha_{\mathrm{f}} / \alpha_{\mathrm{c}}=1$ is called as "surface peak" which corresponds the maximum of $T_{f}$ in Eq. 2a. In the case of $\alpha_{i}<0.18^{\circ}$, the surface peak was clearly observed, suggesting that incident $x$-rays extinguished in the $\mathrm{SiO}_{2}$ and SGOI layers, and the portion of SGOI layer close to the top layer mainly contributed to the Bragg diffraction.

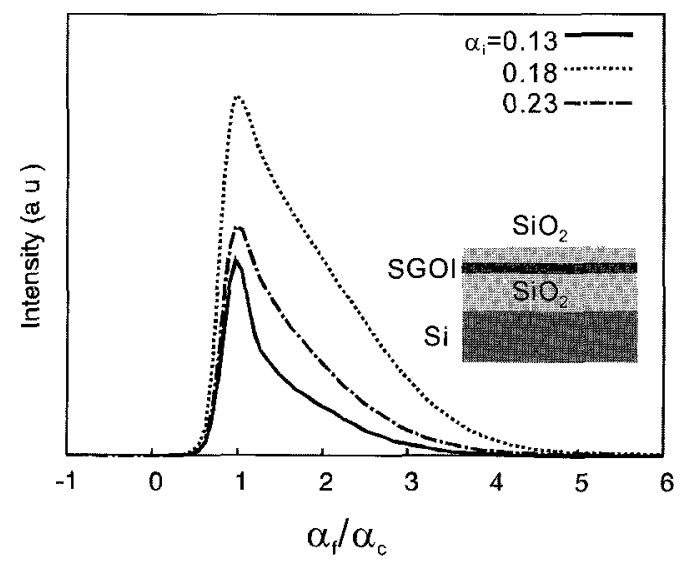

Fig. 2. The SGOI(220) diffraction profiles along $q_{z}$ direction with various incident angles. Horizontal axis is normalized with the critical angle of SGOI layers.

The intensity of surface peak becomes larger with increasing incident angles and decreases again at $\alpha_{i}>\alpha_{c}$. This shows that contribution of deep portion of SGOI layers increases when both $\alpha_{i}$ and $\alpha_{f}$ are larger than $\alpha_{c}$. 
The right shoulders of the surface peak are different in three incident angles, suggesting the inhomogeneity of Ge portion in the SGOI layers.

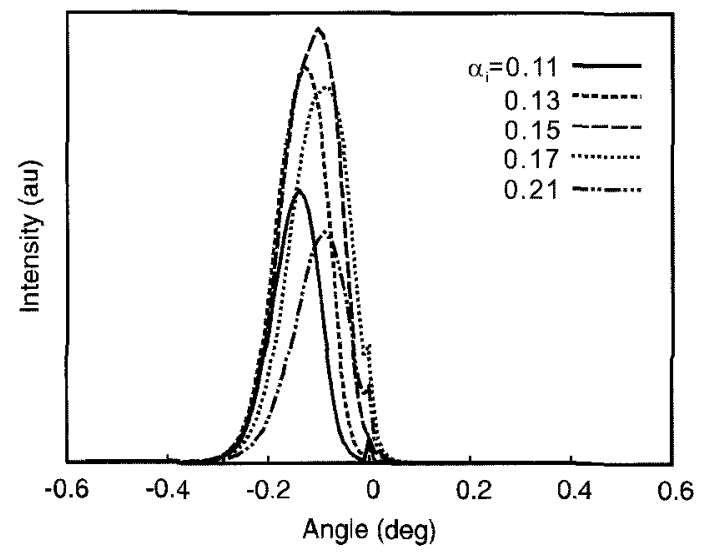

Fig. 3. (220) Bragg diffraction with various incident angles. Differences between the peak positions of SGOI and that of a $\mathrm{Si}$ substrate increase with decreasing incident angles, suggesting Ge portions at the surface is larger than the average one.

To evaluate the strains parallel to the surface, (220) diffractions of SGOI were analyzed. Figure 3 shows the (220) profiles measured at $\alpha_{f}=\alpha_{c}$ with various incident angles. Horizontal axis shows the relative $\Delta \theta$ angles from Si (220) diffractions. Obviously, differences between SGOI and Si substrate peaks become smaller with increasing incident angles, showing the decrease of Ge fraction in the deep part of the SGOI layer. Since large difference of peak position corresponds to large Ge fraction, it suggests that Ge portion decreases from the surface to the bottom

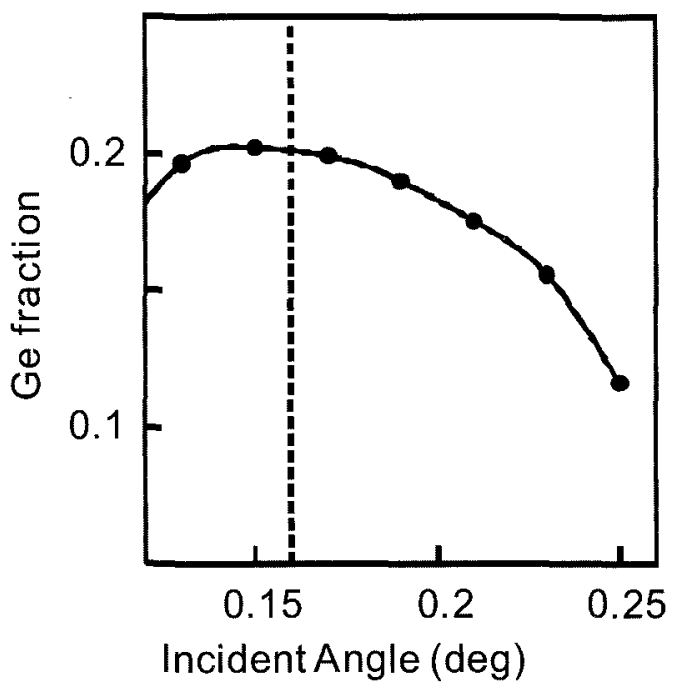

Fig. 4. Ge fraction dependence on the incident angles. To calculate Ge fraction, fully relaxed SGOI layer is assumed.

Since (004) diffraction of SGOI is very weak and wide, it is assumed that SiGe in SGOI layer is fully relaxed, and the Ge fraction is determined from the peak shift with using this assumption. Figure 4 shows the calculated $\mathrm{Ge}$ fraction for each incident angle. When the incident angle is smaller than the critical angle of $\mathrm{SiO}_{2}$, shown as dotted line in Fig. 4, Ge fractions of SGOI is about 0.2 and monotonically decreases with increasing incident angles. The decease is more clearly observed in the region larger than 0.23 degrees which corresponds to the SiGe critical angle, suggesting Ge segregation at the top interface between $\mathrm{SiO}_{2}$ and SGOI. This phenomenon is consistent with the Ge-alloy oxidization mechanism which reported that the Ge concentrated layer was formed between Ge layer and oxidized layer [20].

\section{CONCLUSION}

The Ge fraction in the very-thin SGOI layers was evaluated using the TER-XD technique. The result shows that the Ge segregation layer was formed between the $\mathrm{SiO}_{2}$ and SGOI layer, and $\mathrm{Ge}$ fraction decreased from the $\mathrm{SiO}_{2} / \mathrm{SGOI}$ interface, which is consistent with the previous investigation of $\mathrm{Ge}$ oxidization mechanism. These features will decrease the performance of SGOI-based electron devices and suggest additional fabrication processes, such as extra annealing, are required to improve crystalline quality of SGOI substrates.

\section{ACKNOWLEDGEMENT}

A part of this work was done at SPring- 8 with approvals 2004B0031-ND1d-np, R05A46XU-0032N, and 2005B0936 of the Japan Synchrotron Radiation Research Institute.

\section{REFERENCES}

[1] J. Welser, J. L. Hoyt, S. Takagi, and J. F. Gibbons, Tech. Dig. Int. Electron Devices Meet. 1994, 373-376 (1994).

[2] G. Abstreiter, H. Brugger, T. E. Wolf, H. Jorke, and H. J. Herzog, Phys. Rev. Lett., 54, 2441-2444 (1985).

[3] D. K. Nayak, J. C. S. Woo, J. S. Park, K. L. Wang, and K. P. MacWilliams, Appl. Phys. Lett. 62, 2853-2855 (1993).

[4] T. Mizuno, S. Takagi, N. Sugiyama, H. Satake, A. Kurobe, and A. Toriumi, IEEE Electron Device Lett., EDL-21, 230-232 (2000).

[5] Z. Y. Cheng, M. T. Currie, C. W. Leitz, G. Taraschi, E. A. Fitzgerald, J. L. Hoyt, and D. A. Antoniadas, IEEE Electron Device Lett. 22, 321-323 (2001).

[6] L. J. Huang, J. O. Chu, S. A. Goma, C. P. D'Emic, S. J. Koester, D. F. Canaperi, P. M. Mooney, S. A. Cordes, J . L. Speidell, R. M. Anderson, and H. S. P. Wong, IEEE Electron Device Lett., 49, 1566-1571 (2002).

[7] T. Tezuka, M. Sugiyama, T. Mizuna, and S. Takagi, IEEE Trans. Electron Devices, 50, 1328-1333 (2003).

[8] Y. Ishikawa, N. Shibata, and S. Fukatsu, Appl. Phys. Lett. 75, 983-985 (1999).

[9] S. Fukatsu, Y. Ishikawa, T. Saito, and N. Shibata, Appl. Phys. Lett. 72, 3485-3487 (1998).

[10] L. J. Huang, J. O. Chu, D. F. Canaperi, C. P. D'Emic, R. M. Anderson, S, J. Koester, and H. S. P. 
Wong, Appl. Phys. Lett. 78, 1267-1269 (2001).

[11] F. Y. Huang, M. A. Chu, M. O. Tanner, K. L. Wang, G. D. U'Ren, and M. S. Goorsky, Appl. Phys. Lett. $76,2680-2682(2000)$.

[12] T. Tezuka, N. Sugiyama, T. Mizuno, M. Suzuki, and S. Takagi, Jpn. J. Appl. Phys. 40, 2866-2874 (2001).

[13] H. Yin, K. D. Hobart, F. J. Kub, S. R. Shieh, T. S. Duffy, and J. C. Sturm, Appl. Phys. Lett., 82, 3853-3855 (2003).

[14] J. C. Tsang, P. M. Mooney, F. Dacol, and J. O. Chu, J. Appl. Phys. 75, 8098-8097 (1993).

[15] W. J. Bartels, and W. Nijman, J. Cryst. Growth, 44, 518-525 (1978).

[15] M. Fatemi and R. E. Stahlbush, Appl. Phys. Lett. $58,825-827$ (1991).

[16] J. P. Dismukes, L. Ekstrom and R. J. Paff, J. Phys. Chem., 68, 3021-3027 (1964).

[17] U. Pietsch, H. Metzger, S. Rugel, B. Jenichen and I. K. Robinson, J. Appl. Phys., 74, 2381-2387 (1993).

[18] D. Rose, U. Pietsch and W. Zeimer, J. Appl. Phys., 81, 2601-2606 (1997).

[19] S. K. Sinha, E. B. Sirota, S. Garoff and H. B. Stanley, Phys. Rev. B, 38, 2297-2311 (1988).

[20] F. K. LeGoues, R. Rosenburg, T. Nguyen, F. Himpsel, and B. S. Meyerson, J. Appl. Phys., 65, 1724-1728 (1989).

(Recieved December 10, 2007 ; Accepted February 23, 2008) 